PROCEEDINGS OF THE

AMERICAN MATHEMATICAL SOCIETY

Volume 105, Number 4, April 1989

\title{
THE RANK IN HOMOGENEOUS SPACES OF NONPOSITIVE CURVATURE
}

\author{
MARIA J. DRUETTA
}

(Communicated by David J. Ebin)

\begin{abstract}
Given a solvable and simply connected Lie group $G$ with Lie algebra $g$ and a left invariant metric of nonpositive curvature without flat factor, we prove that $\operatorname{rank}(G) \leq \operatorname{dim} a$, where $a$ is the orthogonal complement of $[g, g]$ in $g$. In particular, if $H$ is a simply connected homogeneous space of nonpositive curvature satisfying the visibility axiom then $H$ has rank one.
\end{abstract}

\section{INTRODUCTION}

Let $G$ be a solvable and simply connected Lie group with a left invariant metric of nonpositive curvature. If $g$ is the Lie algebra of $G$, then $g=$ $[g, g] \oplus a$ where $a$, the orthogonal complement of $[g, g]$ in $g$ with respect to the metric, is an abelian subalgebra of $g$.

The rank of $G(\operatorname{rank}(G))$ is defined as the minimum of the dimensions of the spaces of parallel Jacobi vector fields along the geodesics through the identity of $G$. This definition coincides with the usual one in the symmetric case.

In this paper we show that if $G$ does not have de Rham flat factor, then $\operatorname{rank}(G)$ is at most $\operatorname{dim} a$ (Theorem 1.3). This bound for $\operatorname{rank}(G)$ is the best possible since for symmetric $G$, it coincides with $\operatorname{dim} a$ (Remark 1.4).

As a consequence, we obtain that if $H$ is a simply connected homogeneous space of nonpositive curvature satisfying the visibility axiom then $H$ has rank one (Corollary 1.6). This fact was proved in [5, Theorem 2.6] for $\operatorname{dim} H \leq 4$.

Finally, we show that the strict inequality in Theorem 1.3 may occur. In particular, we obtain examples of rank 1-homogeneous spaces of nonpositive curvature having planes of zero curvature (hence, they are not symmetric). Moreover, they do not satisfy the visibility axiom.

\section{Preliminaries}

Let $H$ be a simply connected homogeneous Riemannian manifold of nonpositive curvature $(K \leq 0)$. If $\gamma$ is a unit geodesic in $H, \operatorname{rank}(\gamma)$ is defined

Received by the editors March 15, 1987.

1980 Mathematics Subject Classification (1985 Revision). Primary 53C30; Secondary 53C25.

Key words and phrases. Homogeneous spaces, nonpositive curvature, rank, visibility axiom.

Supported in part by CONICOR. 
to be the dimension of the vector space of parallel Jacobi vector fields on $\gamma$. The rank of $H$ (denoted by $\operatorname{rank}(H))$ is the minimum of $\operatorname{rank}(\gamma)$ over all unit geodesic $\gamma$ of $H$ such that $\gamma(0)=p$ for some $p$ in $H$. This definition was introduced in [3] ( $H$ not necessarily a homogeneous space) and it coincides with the usual one if $H$ is a symmetric space (see [5, Preliminaries]). It is clear that $\operatorname{rank}(H) \geq 1$ and $\operatorname{rank}(H)=\operatorname{dim} H$ if and only if $H$ is flat.

Being $H$ homogeneous it admits a simply transitive solvable Lie group of isometries (see [1, Proposition 2.5]) and hence $H$ is isometric to a solvable Lie group $G$ with a left invariant metric of nonpositive curvature.

For $G$ as above, $G$ satisfies the visibility axiom if and only if $G$ admits a left invariant metric of negative curvature (see [4, Corollary 2.3]). Hence, if $G$ has sectional curvature $K<0, G$ satisfies the visibility axiom and it also follows, by the definition of rank, that $G$ has rank one.

We recall that if $X, Y, Z \in g$, the Lie algebra of $G$, the Riemannian connection $\nabla$ is given by

$$
2\left\langle\nabla_{X} Y, Z\right\rangle=\langle[X, Y], Z\rangle-\langle[Y, Z], X\rangle+\langle[Z, X], Y\rangle
$$

and the sectional curvature $K$ at $e$, the identity of $G$, is defined by

$$
|X \wedge Y|^{2} K(X, Y)=\langle R(X, Y) Y, X\rangle
$$

where $R(X, Y)=\left[\nabla_{X}, \nabla_{Y}\right]-\nabla_{[X, Y]}$.

In general, in a Lie group $G$, if for any $g \in G, L_{g}$ and $R_{g}$ denote the left and right translations respectively and $I_{g}=L_{g} \circ R_{g^{-1}}$, then the adjoint representation of $G$ defined by $\operatorname{Ad}(g)=\left(d I_{g}\right)_{e}$ satisfies $I_{g}(\exp X)=\exp (\operatorname{Ad}(g) X)$ and $\operatorname{Ad}(\exp X)=\operatorname{Exp}\left(\operatorname{ad}_{X}\right)$ for every $X$ in $g$, where exp: $g \rightarrow G$ is the exponential map of $G$ and Exp denote the exponential map in $g \ell(g)$.

\section{THE RANK OF $G$}

Let $G$ be a solvable and simply connected Lie group $G$ with a left invariant metric of nonpositive curvature. If $g$ is the Lie algebra of $G$, then $g=$ $[g, g] \oplus a$ where $a$, the orthogonal complement of $[g, g]$ with respect to the metric, is an abelian subalgebra of $g$. (see [1, Theorem 5.2].)

If $g^{\prime c}$ is the complexification of $g^{\prime}=[g, g]$ then we have a decomposition in direct sum, $g^{\prime c}=\sum_{\lambda} g_{\lambda}^{\prime c}$, where $g_{\lambda}^{\prime c}=\left\{U \in g^{\prime c}:\left(\operatorname{ad}_{H}-\lambda(H) I\right)^{k} \cdot U=0\right.$ for some $k \geq 1$ and for all $H \in a\}$ is the associated root space for the root $\lambda \in\left(a^{*}\right)^{c}$ under the abelian action of $a$ on $g^{\prime}$. If $\lambda=\alpha \pm i \beta$ is a root of $a$ in $g^{\prime}$ (that is $g_{\lambda}^{\prime c} \neq 0$ ), the generalized root space is defined by $g_{\alpha, \beta}^{\prime}=g_{\alpha,-\beta}^{\prime}=$ $g^{\prime} \cap\left(g_{\lambda}^{\prime c} \oplus g_{\lambda}^{\prime \prime}\right)$ and $g^{\prime}$ is the direct sum of the generalized root spaces $\mathscr{g}_{\alpha, \beta}^{\prime}$.

We assume that $G$ has no de Rham flat factor; it follows from [2, Theorem 4.6] that the factors $g_{0}=\sum g_{0, \beta}$ and $a_{0}=\{H \in a: \alpha(H)=0$ for all roots $\alpha+i \beta\}$ are zero. Then $g^{\prime}=\sum_{\alpha \neq 0} g_{\alpha, \beta}^{\prime}$.

The following lemma is the key for the proof of Theorem 1.3. 
Lemma 1.1. If $H \in a$ satisfies $\alpha(H)>0$ for $\alpha+i \beta$ a root then

$$
\lim _{t \rightarrow+\infty} \operatorname{Exp}\left(-t \operatorname{ad}_{H}\right) X=0 \text { for all } X \in g_{\alpha, \beta}^{\prime} \text {. }
$$

Proof. Let $\lambda=\alpha+i \beta$ be a root of $a$ in $g^{\prime}$. By definition of $g_{\lambda}^{\prime c}, N=$ $\left.\left(\operatorname{ad}_{H}-\lambda(H) I\right)\right|_{\mathcal{g}_{i}^{\prime \prime}}$ is a nilpotent operator on $g_{\lambda}^{\prime c}$. Then $\left.\operatorname{ad}_{H}\right|_{\mathcal{g}_{i}^{\prime \prime}}=\lambda(H) I+N$ and $\left.\operatorname{Exp}\left(-t \operatorname{ad}_{H}\right)\right|_{\mathscr{g}_{i}^{\prime \prime}}=e^{-t \lambda(H)} \operatorname{Exp}(-t N)$; since $\left|e^{-i t \beta(H)}\right|=1$ it follows that $\left.\lim _{t \rightarrow+\infty} \operatorname{Exp}\left(-t \operatorname{ad}_{H}\right)\right|_{\mathscr{g}_{i}^{\prime \prime}}=0$ if and only if $\lim _{t \rightarrow+\infty} e^{-t \alpha(H)} \operatorname{Exp}(-t N)=0$ in $G l\left(g_{\lambda}^{\prime c}\right)$. We compute this limit in each matricial coordinate $(i j)$. Since $N$ is nilpotent,

$$
\operatorname{Exp}(-t N)=\sum_{k=0}^{s}(-1)^{k} \frac{t^{k}}{k !} N^{k}\left(N^{s+1}=0\right)
$$

and

$$
\operatorname{Exp}(-t N)_{i j}=\sum_{k=0}^{s}(-1)^{k} \frac{t^{k}}{k !}\left(N^{k}\right)_{i j}=P_{i j}^{s}(t)
$$

is a polynomial in $t$ of degree $s \geq 0$. Then, $\lim _{t \rightarrow+\infty} e^{-t \alpha(H)}(\operatorname{Exp}(-t N))_{i j}=$ $\lim _{t \rightarrow+\infty} e^{-t \alpha(H)} P_{i j}^{s}(t)=0$ since $\alpha(H)>0$.

Hence, $\lim _{t \rightarrow+\infty} \operatorname{Exp}\left(-t \operatorname{ad}_{H}\right) U=0$ for all $U \in g_{\lambda}^{\prime c}$ such that $\lambda=\alpha+i \beta$ is a root and consequently, $\lim _{t \rightarrow+\infty} \operatorname{Exp}\left(-t \operatorname{ad}_{H}\right) X=0$ for all $X \in g_{\alpha, \beta}^{\prime}$.

A Jacobi vector field $J$ on a geodesic $\gamma$ is said to be stable if there exists a constant $c>0$ such that $|J(t)| \leq c$ for all $t \geq 0$. We recall that if $\gamma$ is a geodesic of $G$, for every tangent vector $v$ at $\gamma(0)$ there exists a unique stable Jacobi vector field $J$ on $\gamma$ such that $J(0)=v$ (see [6, Lemma 2.2]). It is obvious that every parallel vector field on a geodesic $\gamma$ is stable.

Lemma 1.2. Let $H \in a$ be such that $\alpha(H)>0$ for all roots $\alpha+i \beta$ (such an $H$ exists by [1, Proposition 5.6] since $G$ has no flat factor). Then the stable Jacobi vector field $J$ on the geodesic $\gamma_{H}(t)=\exp t H$ with $J(0)=X \in g^{\prime}$ is given by $J(t)=\tilde{X}_{\exp t H}$, where $\tilde{X}$ is the right invariant vector field on $G$ such that $\tilde{X}_{e}=X$.

Proof. It follows from Lemma 1.1 since $\tilde{X}$ is a Jacobi field on $\gamma_{H}$ and $\left|\tilde{X}_{\exp t H}\right|$ $=|\operatorname{Ad}(\exp -t H) X|=\left|\operatorname{Exp}\left(-t \operatorname{ad}_{H}\right) X\right|$.

Theorem 1.3. Let $G$ be a solvable and simply connected Lie group with a left invariant metric of nonpositive curvature. If $G$ has no de Rham flat factor then $1 \leq \operatorname{rank}(G) \leq \operatorname{dim} a$.

Proof. Let $a^{\prime}=\{H \in a: \alpha(H)>0$ for all roots $\alpha+i \beta\}$. We will show that if $H \in a^{\prime}$ then there is no parallel Jacobi vector field $J$ on $\gamma_{H}$ with $J(0) \neq 0$ in $g^{\prime}$. In fact, if such a $J$ exists, $J$ is a stable vector field on $\gamma_{H}$ with $J(0)=X \in g^{\prime}$ and from Lemma 1.2, $J(t)=\tilde{X}_{\exp t H}$ for all $t \in \mathbf{R}$. This is a contradiction since $\lim _{t \rightarrow+\infty}\left|\tilde{X}_{\exp t H}\right|=0$ and $|J(t)|=|X|$ for all $t \in \mathbf{R}$. 
Therefore, $J$ is a parallel vector field on $\gamma_{H}$ if and only if $J(t)=\left(d L_{\exp t H}\right)_{e} Z$ with $Z \in a\left(\nabla_{H} Z=0\right)$; hence the dimension of the space of parallel Jacobi vector fields on $\gamma_{H}$ equals $\operatorname{dim} a$ and consequently $\operatorname{rank}\left(\gamma_{H}\right)=\operatorname{dim} a$ for all $H \in a^{\prime}$. Hence, $\operatorname{rank}(G) \leq \operatorname{dim} a$.

We note that if $G$ admits de Rham flat factor then $\operatorname{rank}(G) \leq \operatorname{dim} a+\operatorname{dim} \mathscr{g}_{0}$ (see [2, Theorem 4.6]).

Remark 1.4. This bound for $\operatorname{rank}(G)$ is the best possible since in the symmetric case $(\nabla R=0), \operatorname{rank}(G)$ coincides with $\operatorname{dim} a$. In fact, $G$ being a symmetric space of noncompact type ( $G$ has no flat factor) it follows from $[7, \S 6$, Chapter V] that $\operatorname{rank}(G)$ is the maximal dimension of a flat Euclidean isometrically imbedded in $G$ as a complete totally geodesic submanifold. Consequently $\operatorname{rank}(G) \geq \operatorname{dim} a$, since $\exp (a)$ satisfies the above conditions (see $[4, \S 2])$. Hence, Theorem 1.3 implies that $\operatorname{rank}(G)=\operatorname{dim} a$.

Theorem 1.5. Let $G$ be a solvable and simply connected Lie group with a left invariant metric of nonpositive curvature. If $G$ satisfies the visibility axiom then $G$ has rank one.

Proof. It is a direct corollary of Theorem 1.3 , since if $G$ satisfies the visibility axiom then $G$ has no flat factor and $\operatorname{dim} a=1$ (see [4, Theorem 2.3]).

Corollary 1.6. If $H$ is a Riemannian simply connected homogeneous space of nonpositive curvature satisfying the visibility axiom then $H$ has rank one. In particular, if $H=G / T$ admits a $G$-invariant metric of negative curvature, $H$ has rank one.

Proof. The first assertion is immediate and the last one follows from [4, Theorem 2.1].

\section{EXAMPLE}

In this section, we exhibit a Lie group $G$ with a left invariant metric of nonpositive curvature such that $\operatorname{rank}(G)=1$ and $\operatorname{dim} a=2$, thus showing that strict inequality in Theorem 1.3 occurs. In this example the commutator subalgebra $g^{\prime}$ is not abelian, in contrast to the case presented in [5, Example 3.3].

First we give a formula for the sectional curvature for a special case.

Lemma 2.1. Let $g$ be a solvable Lie algebra with an inner product $\langle$,$\rangle such$ that $a$, the orthogonal complement of $g^{\prime}$ is abelian. If $\mathrm{ad}_{H_{y^{\prime}}}$ is symmetric with respect to $\langle$,$\rangle for all H \in a$, then

$$
\begin{aligned}
\langle R(X+H, Y+T)(Y+T), X+H\rangle & =\langle R(X, Y) Y, X\rangle-|[H, Y]-[T, X]|^{2}-\langle[H, Y]-[T, X],[X, Y]\rangle \\
& +\langle[[H, Y]-[T, X], X], Y\rangle-\langle[[H, Y]-[T, X], Y], X\rangle
\end{aligned}
$$

for all $X, Y \in g^{\prime}, H, T \in a$. 
Proof. Let $X, Y \in g^{\prime}, H, T \in a$. Since $R(T, H)=0$ and $\nabla_{H}=0$ for all $H, T \in a$, by the definition of $R$ we have,

$$
\begin{aligned}
\langle R(X+H, Y+T)(Y+T), X+H\rangle= & \langle R(X, Y) Y, X\rangle+\langle R(X, T) T, X\rangle \\
& +\langle R(H, Y) Y, H\rangle+2\langle R(H, Y) Y, X\rangle \\
& +2\langle R(X, T) Y, X\rangle+2\langle R(H, Y) T, X\rangle \\
= & \langle R(X, Y) Y, X\rangle-|[T, X]|^{2} \\
& -|[H, Y]|^{2}-2\left\langle\nabla_{[H, Y]} Y, X\right\rangle \\
& -2\left\langle\nabla_{[T, X]} X, Y\right\rangle-2\left\langle\nabla_{[H, Y]} T, X\right\rangle .
\end{aligned}
$$

By using the connection formula,

$$
\begin{aligned}
& 2\left\langle\nabla_{[H, Y]} Y, X\right\rangle=\langle[[H, Y], Y], X\rangle-\langle[Y, X],[H, Y]\rangle+\langle[X,[H, Y]], Y\rangle \\
& 2\left\langle\nabla_{[T, X]} X, Y\right\rangle=\langle[[T, X], X], Y\rangle-\langle[X, Y],[T, X]\rangle+\langle[Y,[T, X]], X\rangle \\
& 2\left\langle\nabla_{[H, Y]}^{T, X\rangle}=\langle[[H, Y], T], X\rangle-\langle[T, X],[H, Y]\rangle,\right.
\end{aligned}
$$

We get,

$$
\begin{aligned}
\langle R(X+ & H, Y+T)(Y+T), X+H\rangle \\
= & \langle R(X, Y) Y, X\rangle-|[T, X]|^{2}-|[H, Y]|^{2}+2\langle[T, X],[H, Y]\rangle \\
& -\langle[[H, Y]-[T, X], Y], X\rangle \\
& +\langle[[H, Y]-[T, X], X], Y\rangle-\langle[H, Y]-[T, X],[X, Y]\rangle
\end{aligned}
$$

and the formula follows.

Example 2.2. Let $g$ be the Lie algebra of dimension five that is generated by $\left\{e_{1}, e_{2} \ldots e_{5}\right\}$ and Lie bracket

$$
\begin{gathered}
{\left[e_{1}, e_{2}\right]=\varepsilon e_{3}, \quad \varepsilon>0} \\
{\left[e_{1}, e_{3}\right]=\left[e_{2}, e_{3}\right]=\left[e_{4}, e_{5}\right]=0} \\
{\left[e_{4}, e_{1}\right]=\gamma e_{1}, \quad\left[e_{4}, e_{2}\right]=-\gamma e_{2}, \quad\left[e_{4}, e_{3}\right]=0} \\
{\left[e_{5}, e_{1}\right]=\alpha e_{1}, \quad\left[e_{5}, e_{2}\right]=\beta e_{2}, \quad\left[e_{5}, e_{3}\right]=(\alpha+\beta) e_{3}}
\end{gathered}
$$

where $\gamma \neq 0, \alpha>0, \beta>0$ are chosen such that $\gamma^{2}-\alpha \beta<0$.

Note that $g^{\prime}$ is nonabelian, it is spanned by $\left\{e_{1}, e_{2}, e_{3}\right\}$ and its center $z$ is $\mathbf{R} e_{3}$.

Let $\langle$,$\rangle be the inner product in g$ such that $\left\langle e_{i}, e_{j}\right\rangle=\delta_{i j} \quad i, j=1 \ldots 5$ and observe that $\left.\operatorname{ad}_{e_{4}}\right|_{g^{\prime}},\left.\operatorname{ad}_{e_{5}}\right|_{g^{\prime}}$ are symmetric with respect to $\langle$,$\rangle .$

For each $\varepsilon \geq 0$ let $\left(g_{\varepsilon},\langle\rangle,\right)$ denote the Lie algebra with the same inner product as the one in $g$ and the same Lie bracket except for $\left[e_{1}, e_{2}\right]_{\varepsilon}=\varepsilon e_{3}$. We note that $g^{\prime}=g_{\varepsilon}^{\prime}=g_{0}^{\prime}\left(g=g_{\varepsilon}=g_{0}\right)$ and $a=a_{\varepsilon}$ as vector spaces. 
By a straightforward computation, using the connection and the curvature formulas we get

$$
\begin{gathered}
\nabla_{e_{1}} e_{1}=\gamma e_{4}+\alpha e_{5}, \quad \nabla_{e_{1}} e_{2}=\frac{1}{2} \varepsilon e_{3}, \quad \nabla_{e_{1}} e_{3}=-\frac{1}{2} \varepsilon e_{2} \\
\nabla_{e_{2}} e_{2}=-\gamma e_{4}+\beta e_{5}, \quad \nabla_{e_{2}} e_{3}=\frac{1}{2} \varepsilon e_{1}, \quad \nabla_{e_{3}} e_{3}=(\alpha+\beta) e_{5} \\
\nabla_{e_{1}} e_{4}=-\gamma e_{1}, \quad \nabla_{e_{1}} e_{5}=-\alpha e_{1}, \quad \nabla_{e_{2}} e_{4}=\gamma e_{2} \\
\nabla_{e_{2}} e_{5}=-\beta e_{2}, \quad \nabla_{e_{3}} e_{4}=0, \quad \nabla_{e_{3}} e_{5}=-(\alpha+\beta) e_{3} \\
K_{\varepsilon}\left(e_{1}, e_{2}\right)=-\frac{3}{4} \varepsilon^{2}+\gamma^{2}-\alpha \beta \\
K_{\varepsilon}\left(e_{2}, e_{3}\right)=\frac{1}{4} \varepsilon^{2}-\beta(\alpha+\beta) \\
K_{\varepsilon}\left(e_{1}, e_{3}\right)=\frac{1}{4} \varepsilon^{2}-\alpha(\alpha+\beta)
\end{gathered}
$$

(3) If $X=a e_{1}+b e_{2}$ and $Y$ in $z^{\perp}$ (the orthogonal complement of $z$ in $g^{\prime}$ ) is independent with $X$, up to a positive constant,

$$
K_{\varepsilon}\left(X, Y+c e_{3}\right)=|X \wedge Y|^{2} K_{\varepsilon}\left(e_{1}, e_{2}\right)+c^{2}\left[a^{2} K_{\varepsilon}\left(e_{1}, e_{3}\right)+b^{2} K_{\varepsilon}\left(e_{2}, e_{3}\right)\right] .
$$

Now, if $X, Y \in g^{\prime}, H, T \in a$ and $\{X+H, Y+T\}$ are orthonormal vectors in $g$, it follows from Lemma 2.1 that

(4) $K_{\varepsilon}(X+H, Y+T)=\left\langle R_{\varepsilon}(X, Y) Y, X\right\rangle-|[H, Y]-[T, X]|^{2}+\varepsilon f(X, Y, H, T)$

where $R_{\varepsilon}$ is the curvature tensor for $\left(g_{\varepsilon},\langle\rangle,\right)$ and $f$ is a continuous function of $X, Y, H, T$. Moreover, if $G_{5,2}(g)$ is the Grassmann manifold of 2planes of $g$, the curvature function $(\varepsilon, \pi) \rightarrow K_{\varepsilon}(\pi)$ is uniformly continuous for $0 \leq \varepsilon \leq 1, \pi \in G_{5,2}(g)$. In fact; since any 2-plane $\pi \subset g$ is spanned by orthonormal vectors $\{X+H, Y+T\}$ with $X \in z^{\perp}$, by using (3) and (4) it is an easy computation to show that $K_{\varepsilon}(\pi)$ is continuous; $G_{5,2}(g)$ being compact, the assertion follows.

As $\varepsilon \rightarrow 0,\left(g_{\varepsilon},\langle\rangle,\right)$ tends to a well defined limit algebra $\left(g_{0},\langle\rangle,\right)$ such that $g_{0}^{\prime}$ is abelian. Then,

(i) If $X, Y \in g_{0}^{\prime}, H, T \in a$ and $\{X+H, Y+T\}$ are orthonormal vectors,

$$
K_{0}(X+H, Y+T)=\left\langle R_{0}(X, Y) Y, X\right\rangle-|[H, Y]-[T, X]|^{2} .
$$

(ii) If $X=a e_{1}+b e_{2}$ and $Y \in z^{\perp}$ is independent with $X$, by the formula given in (3), up to a positive constant,

$$
K_{0}\left(X, Y+c e_{3}\right)=|X \wedge Y|^{2}\left(\gamma^{2}-\alpha \beta\right)-(\alpha+\beta) c^{2}\left(a^{2} \alpha+b^{2} \beta\right)<0 .
$$

In particular, $\left(g_{0},\langle\rangle,\right)$ has sectional curvature $K_{0} \leq 0$.

(iii) Since any 2-plane $\pi \subset g_{0}^{\prime}$ contains a vector in $z^{\perp}$, we have that there exists a number $r>0$ such that $K_{0}(\pi)<-r$ for all 2-plane $\pi \subset g_{0}^{\prime}$. (The curvature function $K_{0}$ is continuous and negative on the Grassmann manifold of 2-planes in $g_{0}^{\prime}$ which is compact.) 
(iv) From the uniform continuity stated above, there exist $\varepsilon_{0}>0$ such that, if $\varepsilon \leq \varepsilon_{0}$ then

$$
K_{\varepsilon}(\pi)<K_{0}(\pi)+r \text { for all } 2 \text {-plane } \pi \subset g \text {. }
$$

Now, let $\pi$ be a 2-plane in $g$ spanned by orthonormal vectors $\{X+H, Y+T\}$ with $X, Y \in g^{\prime}, H, T \in a$. First we assume that $X, Y$ are independent. Hence, by using (i) and (iii),

$$
K_{0}(\pi)=|X \wedge Y|^{2} K_{0}(X, Y)-|[H, Y]-[T, X]|^{2} \leq K_{0}(X, Y)<-r .
$$

Consequently, (iv) implies that,

$$
K_{\varepsilon}(\pi)<K_{0}(\pi)+r \leq K_{0}(X, Y)+r<0 \text { if } \varepsilon \leq \varepsilon_{0} .
$$

If $Y$ is a multiple of $X, K_{\varepsilon}(\pi)=-|[H, Y]-[T, X]|^{2} \leq 0$ (the other terms in (4) are zero).

Hence, if $\varepsilon \leq \varepsilon_{0}\left(g_{\varepsilon},\langle\rangle,\right)$ has sectional curvature $K_{\varepsilon} \leq 0$. Moreover, if $X=a e_{1}+b e_{2}$ with $a \neq 0 \quad b \neq 0$ is a unit vector, then for $Y \in g^{\prime}, T \in a$ such that $\{X, Y+T\}$ are orthonormal we have, if $Y \neq 0, K_{\varepsilon}(X, Y+T)<0((\mathrm{v}))$ if $T \neq 0, K_{\varepsilon}(X, T)=-|[X, T]|^{2}<0$. In fact, $[X, T]=a\left\langle T, \gamma e_{4}+\alpha e_{5}\right\rangle e_{1}+$ $b\left\langle T,-\gamma e_{4}+\beta e_{5}\right\rangle e_{2}$ is nonzero, since $a \neq 0, b \neq 0$ and $\gamma e_{4}+\alpha e_{5},-\gamma e_{4}+\beta e_{5}$ are linearly independent in $a$.

Hence, if $\varepsilon \leq \varepsilon_{0}$ and $G_{\varepsilon}$ is the simply connected Lie group associated to $\left(g_{\varepsilon},\langle\rangle,\right)$, the geodesic $\gamma$ in $G_{\varepsilon}$ with $\gamma^{\prime}(0)=X$ has rank one; therefore, $\operatorname{rank}\left(G_{\varepsilon}\right)=1$.

\section{REFERENCES}

1. R. Azencott and E. Wilson, Homogeneous manifolds with negative curvature. I, Trans. Amer. Math. Soc. 215 (1976), 326-362.

2. __ Homogeneous manifolds with negative curvature. II, Mem. Amer. Math. Soc. no. 178 (1976).

3. W. Ballmann, M. Brin and P. Eberlein, Structure of manifolds of nonpositive curvature. I, Ann. of Math. (2) 122 (1985).

4. M. J. Druetta, Homogeneous riemannian manifolds and the visibility axiom, Geometriae Dedicata 17 (1985), 239-251.

5. __ Visibility and rank one in homogeneous spaces of $K \leq 0$, Trans. Amer. Math. Soc. 304 (1987), 307-321.

6. E. Heintze and Im Hof, Geometry of horospheres, J. Differential Geom. 12 (1977), 481-491.

7. S. Helgason, Differential geometry, Lie groups and symmetric spaces, Academic Press, New York, 1978.

Facultad de Matemática, Astronomía y Física, Universidad Nacional de Córdoba, Valparaiso y R. Martínez, Ciudad Universitaria, 5000 Córdoba, Argentina 